\title{
Diffusion imaging shows abnormalities after blunt head trauma when conventional magnetic resonance imaging is normal
}

\author{
F J Rugg-Gunn, M R Symms, G J Barker, R Greenwood, J S Duncan
}

Correspondence to: Professor J S Duncan, National Society for Epilepsy, Chalfont St Peter, Gerrards Cross, Bucks. SL9 0RJ, UK j.duncan@ion.ucl.ac.uk

Received 26 April 2000 and in revised form 28 July 2000 Accepted 10 November 2000

\begin{abstract}
The investigation and management of patients after head injury must include the accurate and complete identification of cerebral damage. Using diffusion tensor imaging, abnormalities of diffusion in patients with head injuries and unremarkable MRI have been shown for the first time.

(7 Neurol Neurosurg Psychiatry 2001;70:530-533)
\end{abstract}

Keywords: diffusion tensor imaging; head injury; mean diffusivity; fractional anisotropy

Head injury is an important cause of physical and psychological disability. Severe blunt head trauma is associated with concussive and contusional injuries with histopathological changes of oedema, petechial and frank haemorrhage, diffuse axonal injury, and ischaemia. The investigation and management of patients after head injury must include the accurate and complete identification of cerebral damage. This often includes CT acutely and MRI later to more accurately characterise chronic cerebral damage. Diffusion weighted imaging (DWI) has been shown to more accurately define the extent of cerebral damage in a closed head injury model than conventional MRI. ${ }^{1}$ Diffusion tensor imaging, a relatively novel MRI technique, detected more extensive abnormalities than conventional MRI in a
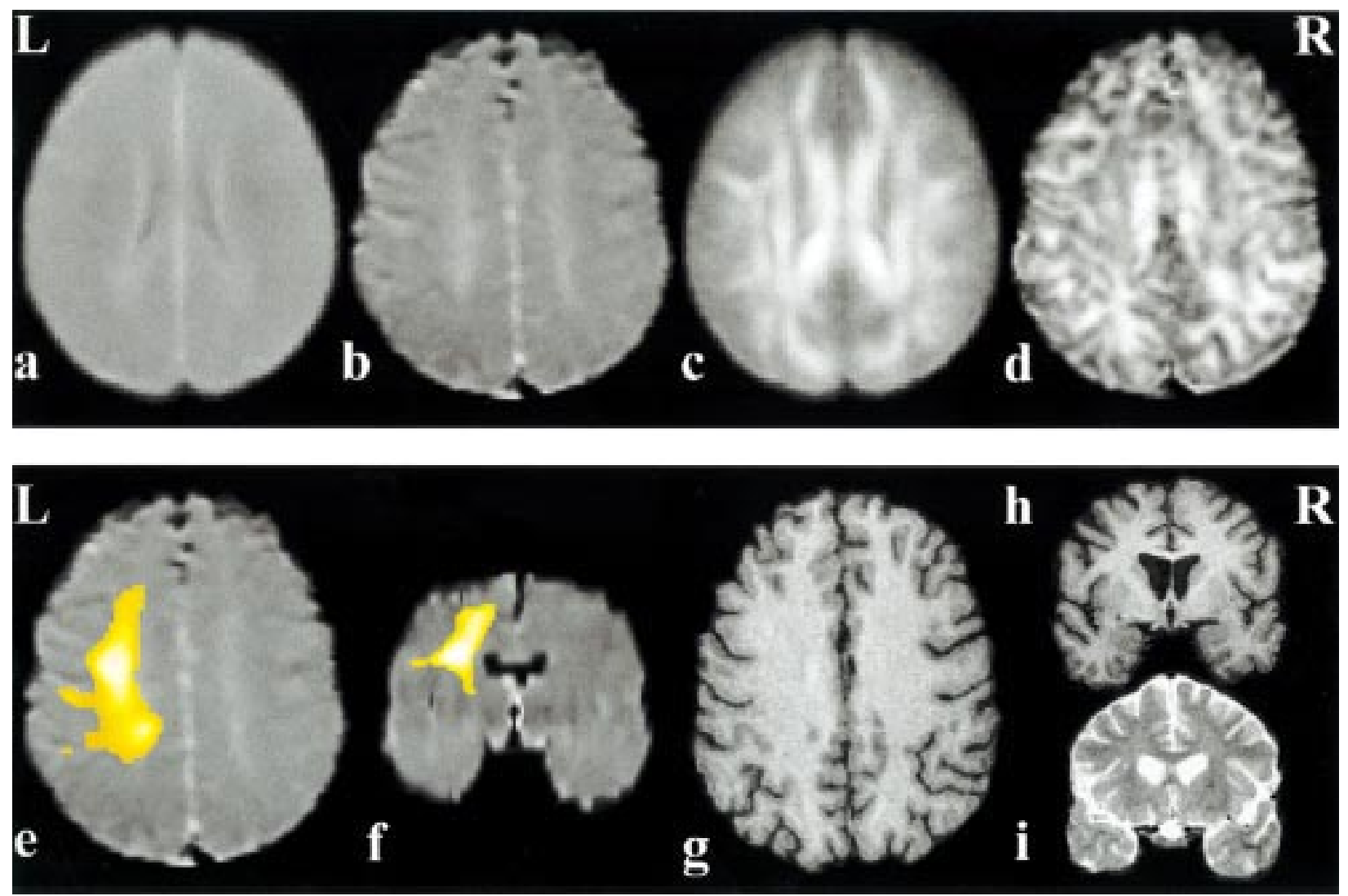

Figure 1 Patient 1. Normalised axial mean diffusivity maps at the same slice position for (a) the averaged 30 control subjects and (b) and (e) the patient. (f) Normalised coronal mean diffusivity map. Normalised axial anisotropy maps at the same slice position for (c) the averaged 30 control subjects and (d) the patient. The difference in signal to noise between the maps is due to averaging of the 30 control subjects. The region of significantly increased mean diffusivity is superimposed on maps $(e)$ and $(f)$. The equivalent slices of the patient's T1 weighted $(g)$ axial and $(h)$ coronal and (i) T2 weighted coronal images show no abnormality. The region in (e) was used for quantitative region of interest analyses of mean diffusivity values. Note that right on the images is the patient's right. 
The MRI Unit, National Society for Epilepsy, and Epilepsy Research Group and NMR Research Unit, University Department of Clinical Neurology, Institute of Neurology, University College London, Queen Square, London WC1N 3BG, UK F J Rugg-Gunn M R Symms

G J Barker J S Duncan

The National Hospital for Neurology and Neurosurgery, University College London, Queen Square, London WC1N 3BG, UK R Greenwood patient with a severe head injury, which corresponded to known neurological deficit. ${ }^{2}$ Using diffusion tensor imaging we have shown, for the first time, abnormalities of diffusion in two patients with head injury and unremarkable conventional MRI.

Diffusion is a random process resulting from the thermal translational motion of molecules. The diffusion displacement distances are comparable with cellular dimensions, so the measurement of water diffusion provides a means of exploring cellular integrity and pathology. Diffusion weighted and diffusion tensor imaging incorporate pulsed magnetic field gradients into a standard MRI sequence resulting in images which are sensitive to the small displacements of water molecules. Diffusion tensor imaging is a development of DWI for which the resulting diffusion parameters, including a quantitative measure of anisotropy, are insensitive to subject positioning and fibre tract alignment within the diffusion gradients of the scanner. Diffusivity is a measurement of the magnitude of diffusion and its directionality is derived from the calculation of anisotropy. ${ }^{3}$ Pathological processes that change the microstructural environment, such as neuronal swelling or shrinkage, increased or decreased extracellular space, and loss of tissue organisation, result in altered diffusion or anisotropy. ${ }^{4}$

\section{Subjects and methods}

PATIENT 1

A 31 year old man had sustained a severe head injury with basal skull fracture, bilateral frontal lobe contusions identified on acute CT, and a 12 day period of unconsciousness after a $3 \mathrm{~m}$ fall from a balcony 18 months previously. Neurological examination disclosed mild pyramidal signs and sensory loss affecting the right arm and leg. Neuropsychological examination showed mild deficits of frontal lobe function. Conventional MRI performed during the same scanning session as the diffusion tensor imaging showed no abnormality other than a few, scattered, non-specific lesions in the region of the white-grey matter interface in both hemispheres.

\section{PATIENT 2}

A 29 year old man had sustained severe head and facial injuries and a 3 day period of unconsciousness after a road traffic accident 11 months previously. His acute CT and conventional MRI after recovery were normal. Neurological examination immediately after the accident showed moderate bilateral tetraparesis, worse on the left. Current neurological examination shows only moderately increased reflexes in the left lower limb. Neuropsychological examination disclosed severe frontal lobe dysfunction and a significant change in personality was also noted. Conventional MRI
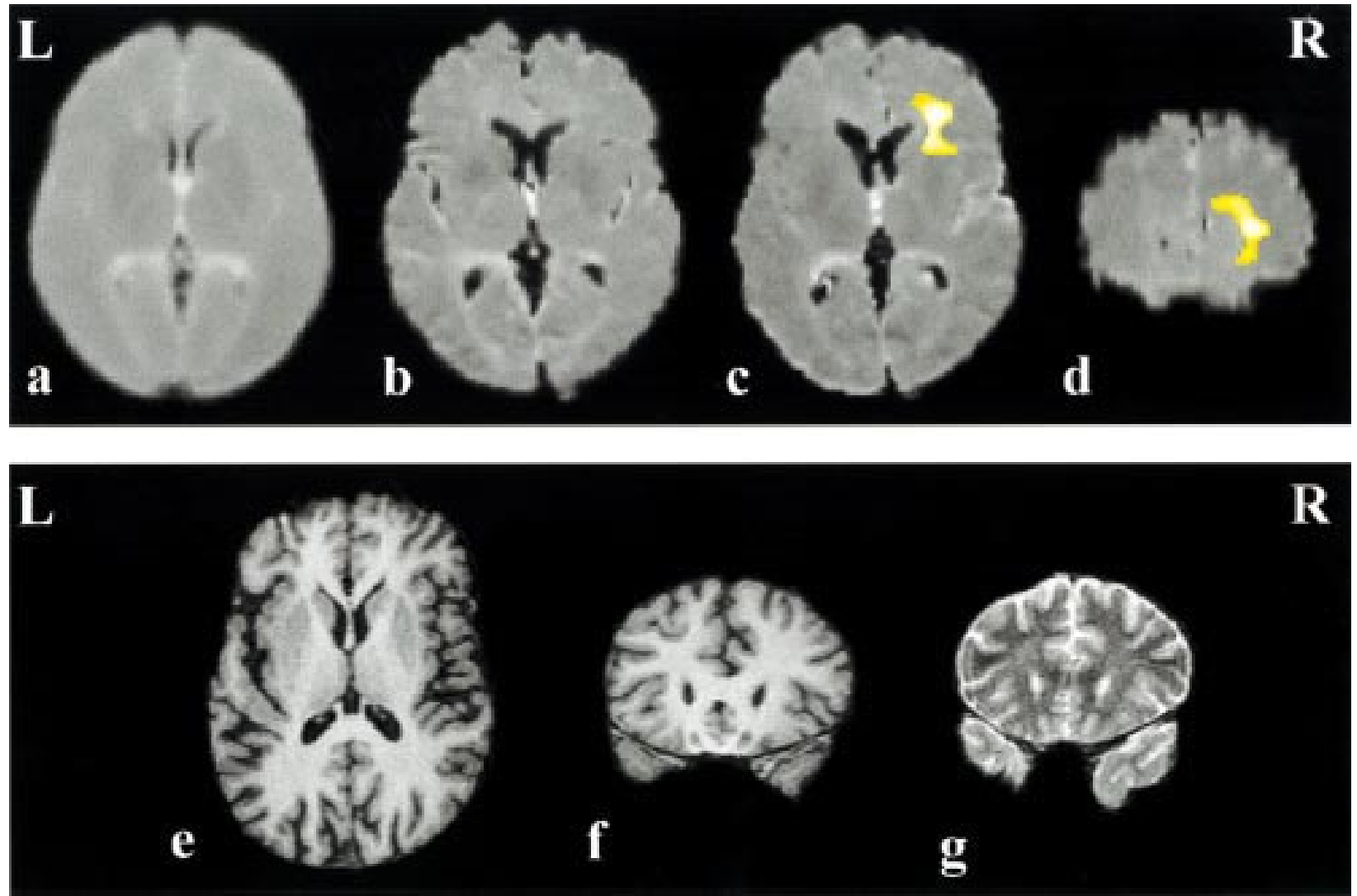

Figure 2 Patient 2. Normalised axial mean diffusivity maps at the same slice position for (a) the averaged 30 control subjects and (b) and (c) the patient. Normalised coronal mean diffusivity map (d). The difference in signal to noise between the maps is due to averaging of the 30 control subjects. The region of significantly increased mean diffusivity is superimposed on maps (c) and (d). The equivalent slices of the patient's T1 weighted axial (e) and coronal $(f)$ and $T 2$ weighted coronal (g) images show no abnormality. The region in (c) was used for quantitative region of interest analyses of mean diffusivity values. Note that right on the images is patient's right. 

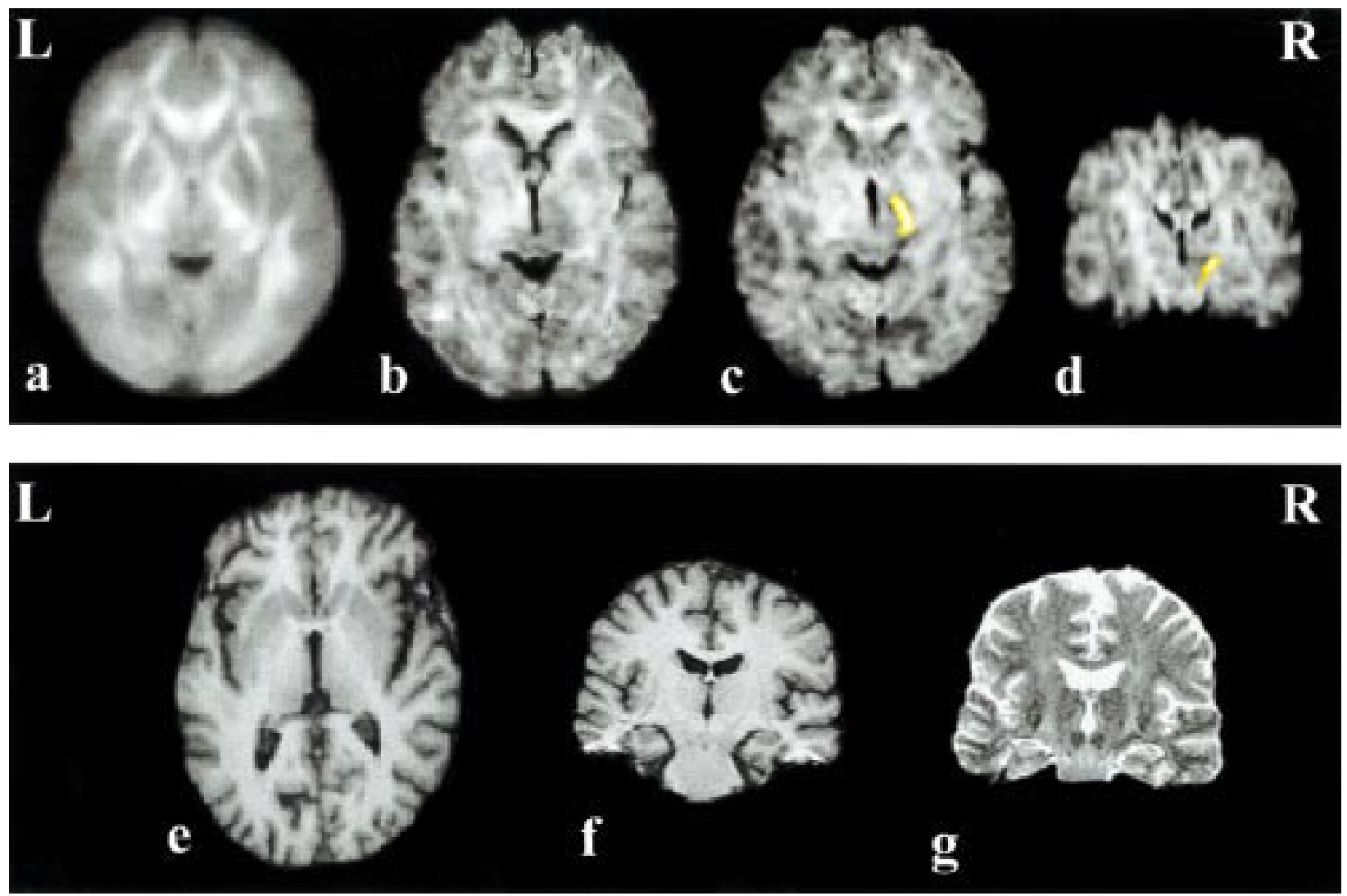

Figure 3 Patient 2. Normalised axial fractional anisotropy maps at the same slice position for the averaged 30 control subjects (a) and the patient (b) and (c). Normalised coronal anisotropy map (d). The difference in signal to noise between the maps is due to averaging of the 30 control subjects. The region of significantly decreased fractional anisotropy is superimposed on maps (c) and (d). The equivalent slices of the patient's T1 weighted (e) axial and $(f)$ coronal and $(g)$ T2 weighted coronal images show no abnormality. The region in (c) was used for quantitative region of interest analyses of fractional anisotropy values. Note that right on the images is patient's right.

performed during the same scanning session as the diffusion tensor imaging was normal.

DIFFUSION TENSOR IMAGING

Diffusion tensor imaging was performed on a 1.5 Tesla GE Horizon MR scanner using single shot CSF suppressed diffusion weighted echoplanar imaging (EPI) (TR/TE/TI $=5000 / 78 /$ $1788 \mathrm{~ms}$, field of view $24 \mathrm{~cm}$, acquisition matrix $96 \times 96$, reconstruction matrix $128 \times 128$, slice thickness $5 \mathrm{~mm}$ ). Pulsed unipolar diffusion gradients were used for diffusion sensitisation $(\delta=28 \mathrm{~ms} ; \Delta=35 \mathrm{~ms})$. Two b values $(0$ and $703 \mathrm{~s} / \mathrm{mm}^{2}$ ) (differing degrees of diffusion weighting) were applied in each of seven directions $(\mathrm{x}, \mathrm{y}, \mathrm{z}, \mathrm{xy}, \mathrm{xz}, \mathrm{yz}, \mathrm{xyz})$ at 13 slice positions. Two interleaved series with nine repeats each were acquired, resulting in 1872 images. The diffusion images were corrected for eddy current induced artefacts by on line scanner software. Diffusion scanning time was 19 minutes. The maps of mean diffusivity and fractional anisotropy were calculated using the method proposed by Pierpaoli and Basser ${ }^{3}$ with in house software. The mean diffusivity map represents the magnitude of the diffusion in each voxel measured in $\mathrm{mm}^{2} / \mathrm{s}$. Each voxel in the fractional anisotropy map represents a value of the anisotropy index, which is a rotationally invariant scalar index of diffusion anisotropy with 0 representing an isotropic medium where there is no directionality to the diffusion and 1.0 representing maximum anisotropy. To allow objective voxel by voxel statistical comparisons to be made between the patient and a group of 30 healthy controls, all diffusion maps were normalised to a standard template. Using SPM, a standard univariate statistical test was applied to every voxel in the image to create a map from which statistical inference was made. ${ }^{5}$ Significant increases or decreases in fractional anisotropy and mean diffusivity were detected at an individual voxel threshold of $p<0.001(p<0.05$ when corrected for multiple comparison).

\section{Results}

PATIENT 1

Comparing the patient's diffusion maps against the similarly normalised maps of 30 healthy control subjects, a significant increase in mean diffusivity was identified in the left corona radiata, extending from the superior aspect of the internal capsule to the subcortical white matter in the frontal and parietal lobes (fig 1). No decreases in mean diffusivity were detected and there were no statistically significant abnormalities of anisotropy.The average mean diffusivity within the abnormal region found in the left corona radiata of the patient was $836 \times 10^{-6} \mathrm{~mm}^{2} / \mathrm{s}$, which was $118 \%$ of the average mean diffusivity within the corresponding regions of the control subjects of $709 \times 10^{-6} \mathrm{~mm}^{2} / \mathrm{s}\left(\mathrm{SD} 23.7 \times 10^{-6} \mathrm{~mm}^{2} / \mathrm{s}\right)$. 
PATIENT 2

Comparing the patient's diffusion maps against the control subjects' maps, a significant increase in mean diffusivity was identified in the right frontal lobe (fig 2), adjacent to the frontal horn of the lateral ventricle and extending into the deep white matter of the right frontal lobe. No decreases in mean diffusivity were detected. The average mean diffusivity within the abnormal region found in the right frontal lobe of the patient was $862 \times 10^{-6} \mathrm{~mm}^{2} / \mathrm{s}$ which was $115 \%$ of the average mean diffusivity within the corresponding regions of the control subjects of $747 \times 10^{-6} \mathrm{~mm}^{2} / \mathrm{s}$ (SD $22.3 \times 10^{-6} \mathrm{~mm}^{2} / \mathrm{s}$ ).

In addition, a significant decrease in anisotropy was detected in the posterior limb of the right internal capsule (fig 3). The average anisotropy within the abnormal region found in the right internal capsule of the patient was 0.47 which was $63 \%$ of the average anisotropy within the corresponding regions of the control subjects of 0.75 (SD 0.04).

\section{Conclusions}

Using diffusion tensor imaging we have shown, for the first time, abnormalities of diffusion in patients with severe head injury and unremarkable conventional MRI. This and other studies ${ }^{2}$ suggest that although abnormalities in mean diffusivity are a more sensitive marker of cerebral damage, quantifying tissue anisotropy yields additional valuable clinical information. To accurately and fully examine the diffusional properties of a tissue, diffusion tensor imaging, with diffusion gradients applied in at least six directions, must be performed. Applying diffusion gradients in only three orthogonal directions leads to an underestimation of anisotropy $^{5}$ which may result in clinically significant findings being overlooked.
In both patients it is likely that the diffusion abnormalities, which are distant from the site of impact, are caused by diffuse axonal injury. Our findings of increased mean diffusivity suggest that there was an expansion of the extracellular space, caused by neuronal or glial cell loss, which was not identified by conventional MRI despite the presence of neurological and neuropsychological symptoms and signs. In addition, a clinically concordant reduction of anisotropy in the internal capsule of patient 2 suggests that there was structural disorganisation and a loss of the parallel fibre arrangement of the major white matter tracts of the internal capsule as a result of the head injury. Again, this was without an identifiable abnormality on conventional MRI.

Our results suggest that diffusion tensor imaging is a useful quantitative imaging method after head injury, and is more sensitive than conventional MRI.

We are grateful to Action Research for supporting this study and FJR-G. MRS is supported by the Brain Research Trust. GJB is supported by the Multiple Sclerosis Society of Great Britain and Northern Ireland. The Glaxo Wellcome Scanner and the MRI unit are supported by the National Society for Epilepsy.

1 Assaf Y, Beit-Yannai E, Shohami E, et al. Diffusion- and T2-weighted MRI of closed-head injury in rats: a time course study and correlation with histology. Magn Reson Imaging 1997;15:77-85.

2 Wieshmann UC, Symms MR, Clark CA, et al. Blunt-head trauma associated with widespread water-diffusion changes. Lancet 1999;353:1242-3.

3 Pierpaoli C, Basser PJ. Toward a quantitative assessment of diffusion anisotropy. Magn Reson Med 1996;36:893-906.

4 Anderson AW, Zhong J, Petroff OAC, et al. Effects of osmotically driven cell volume changes on diffusionweighted imaging of the rat optic nerve. Magn Reson Med 1996;35:162-7.

5 Friston KJ, Holmes AP, Worsley KJ, et al. Statistical parametric maps in functional imaging: a general linear approach. Hum Brain Mapping 1995b;2:189-210. 